\title{
Il turismo, ciliegina sulla torta del Ministero delle politiche agricole alimentari e forestali
}

\author{
Tourism, icing on the cake of the Ministry \\ of agriculture food and forestry policies
}

Dapprima Ministro senza portafoglio per il turismo e lo sport, del secondo "Governo Segni" formato nel febbraio del 1959, Umberto Tupini (degasperiano, già membro dell'Assemblea costituente) fu anche il Ministro che per primo venne nominato a capo del neo-istituito Ministero del turismo e dello spettacolo, sempre nel 1959 (con legge 31 luglio, n. 617).

Allora, il turismo, sbocciato copiosamente nell'Italia della Lancia Aurelia e dei Jolly Hotel, fu considerato meritevole di specifica struttura ministeriale, assieme allo spettacolo (già nel titolo della legge del 1959), allo sport e alle località termali (nel testo della legge).

Con siffatto Ministero, affiancato tra gli altri dal Ministero per i beni culturali a iniziare dal 1974, e dal Ministero dell'ambiente a iniziare dal 1986, i Governi si succedettero, numerosi nelle diverse legislature, sino all'aprile del 1993, quando con voto popolare referendario (18-19 aprile)

* Professore associato nell’Università di Padova - maurizio.malo@unipd.it.

$\checkmark$ Il presente contributo è stato sottoposto a doppio referaggio anonimo - This article has been submitted to double blind peer review.

Rivista italiana di Diritto del turismo, 25-26/2019 - ISSN 2039-9022 ISSNe 2039-9391 DOI: 10.3280/DT2019-025001 
venne abrogata la legge del 1959 istitutiva del Ministero che fu conseguentemente soppresso.

L'ispirazione di quel referendum abrogativo (con altri simmetrici, volti alla soppressione di ministeri) fu dei fautori del regionalismo che, se pure assai più inclini alle esaltazioni padane che alla corretta attuazione dei principi costituzionali sulla forma di stato, diedero una forte spinta nel senso del riconoscimento e della promozione delle autonomie regionali e locali (in base all'articolo 5 della Costituzione, pietra miliare della forma di stato della Repubblica italiana).

Del resto, il disegno costituzionale che implica un numero decisamente limitato di Ministeri, non è teso a ridurre l'importanza di materie in ipotesi prive di espressa organizzazione statale di governo, in quanto attribuite alle Regioni e agli enti locali. All'opposto, esso mira ad una amministrazione pubblica più vicina e sensibile ai caratteri tutt'altro che omogenei del territorio e della società italiana.

E, d'altro canto, ciò non significa negare il bisogno di azioni amministrative del Governo statale, segnatamente in materia di turismo, di coordinamento e promozione. La mancanza di specifica e rigida struttura ministeriale, consente piuttosto di non ingessare al centro, senza giustificazione, funzioni e apparati.

Secondo questa prospettiva, resta ancora ipotizzabile la presenza nel Governo statale di un organo politico espressamente dedicato alle (si presume) poche e tuttavia fondamentali funzioni in materia di turismo, ma non come organo ministeriale in senso formale, bensì come figura organizzativa relativamente snella, presso la Presidenza del Consiglio dei ministri (in ragione del carattere trasversale del turismo, collegato a materie di competenza di diversi dei ministeri), com'è la figura del sottosegretario o la figura del ministro senza portafoglio, con delega ad esercitare funzioni altrimenti del Presidente del Consiglio (dopo la soppressione del Ministero a seguito del risultato referendario del 1993, la delega per il turismo, ad un sottosegretario nel Governo Dini 19951996; ad un ministro senza portafoglio nei Governi "Berlusconi quarto" 2008-2011 e Monti 2011-2013).

Discutibile invece, in punto di diritto costituzionale, la collocazione delle funzioni amministrative statali in materia di turismo, presso un qualsiasi Ministero, attraverso delega (come avvenne nel primo Governo Prodi, 1996-1998, al Ministro dell'industria, commercio e arti- 
gianato) o addirittura mediante (più) stabile modifica legislativa volta a riorganizzare e riqualificare un ministero già istituito, come in un primo tempo il Ministero per i beni e le attività culturali, riordinato nella veste di "Ministero dei beni e delle attività culturali e del turismo" dalla legge n. 71 del 2013; e ora il Ministero delle politiche agricole, alimentari e forestali, riordinato come "Ministero delle politiche agricole, alimentari, forestali e del turismo" (art. 1 del decreto-legge 12 luglio 2018, n. 86, convertito con modificazioni dalla legge 9 agosto 2018, n. $97)^{\dagger}$.

In effetti, il turismo è collegabile a diversi elementi e fenomeni. Se si è orientati agli interessi degli operatori (dagli albergatori agli agenti di viaggio e vari altri) i compiti statali potrebbero riguardare il Ministero dello sviluppo economico. Orientati invece alle modalità del viaggiare, $i$ compiti spetterebbero al Ministero delle infrastrutture e dei trasporti. Ma il turismo è più spesso collegato alle mete del viaggio, che sono principalmente d'interesse artistico, storico e paesaggistico: da qui la più prevedibile collocazione dei compiti presso il Ministero dei beni e delle attività culturali.

Meno prevedibile il turismo presso il Ministero delle politiche agricole e alimentari, ma tutt'altro che implausibile solo a pensare all'agriturismo fattosi addirittura stellato (girasoli o spighe) come il turismo alberghiero (per non dire ora della bolognese Disneyland del cibo, FICO, esposizione permanente da Oscar agroalimentare e... Farinetti). Magari, il prossimo Governo, con altra repentina modifica legislativa, ci

† Nelle more della pubblicazione del presente editoriale, il Consiglio di Stato Cons. St., Sez. cons. atti norm., 28 dicembre 2018, n. 2957 - Affare n. 02107_2018 si è espresso "nel senso che le funzioni non riconducibili alla materia del turismo dovrebbero essere riallocate presso le competenti strutture del Ministero delle politiche agricole alimentari, forestali e del turismo, modificando, per l'effetto, gli artt. 2 e 3 del provvedimento in esame. Spetta alla Presidenza del Consiglio, cui il provvedimento è amministrativamente e politicamente imputato nella forma del d.P.C.M., valutare nella sua responsabile discrezionalità l'opportunità di una rivisitazione del testo nel senso indicato, nonché l'opportunità dell'ulteriore corso del provvedimento con gli attuali contenuti". Il parere è pubblicato in questa Rivista, infra, p. 246. 
offrirà il turismo come contorno del Ministero della salute, memore della grande tradizione termale italiana (in passato ampiamente sostenuta attraverso il sistema degli enti parastatali, mutualistici, dello stato sociale) e memore della medicina preventiva.

Ma "medicina preventiva" vuol dire anche sport, ovvero il "grande fratello" del turismo negli assetti organizzativi dei governi statali. Già unito al turismo nell'originario Ministero (si rammenta che Umberto Tupini fu designato Ministro anche per la sua precedente esperienza come sindaco della Capitale: esperienza preziosa in riferimento alla organizzazione delle Olimpiadi di Roma, del 1960), lo sport compare e scompare nei governi degli ultimi decenni, come un torrente carsico. Accompagnato non al turismo ma allo spettacolo, con "annessione" al Ministero dei beni e attività culturali, lo sport nel primo Governo Prodi (1996-1998) è di competenza di un ministro con portafoglio. Nel secondo Governo Prodi (2006-2008), unito alle politiche giovanili; nel Governo Letta (2013-2014), unito agli affari regionali; e nel Governo Gentiloni (2016-2018), in modo a sé stante, lo sport è delegato ad un ministro senza portafoglio (e nel Governo Renzi, 2014-2016, ad un sottosegretario delegato anche alle politiche di coesione territoriale). Sport e turismo tornano invece assieme (come in origine, già prima dell'istituzione del Ministero), nel Governo Monti (2011-2013), ancora mediante delega ad un ministro senza portafoglio.

Si può dire che queste ondivaghe scelte di assetto governativo statale, che accomunano in particolare il turismo e lo sport, dipendono in certa misura da una interpretazione (corrente in dottrina e nella giurisprudenza costituzionale) delle disposizioni costituzionali sul sistema pubblico composito (Stato, Regioni, enti locali), che enfatizza in modo sfibrante la compartecipazione degli enti nell'azione amministrativa (di indirizzo, promozione, governo, servizio, controllo).

Si può aggiungere, però, che, dopo lunghi anni di prove, di mosse e contromosse volte ad assegnare adeguata collocazione al turismo in seno al Governo statale, e nella consapevolezza del suo assoluto rilievo come forma di salvaguardia e sviluppo del sistema economicosociale italiano, ora nel 2018, a pochi mesi di distanza dalla celebrazione del "turismo da record come baricentro per la ripartenza del Paese" (Rapporto Censis 2017), collocare la materia quale ciliegina sulla torta del Ministero per le politiche agroalimentari, appare soluzione poco 
meditata, inopinatamente settoriale, pur con tutta la simpatia (o la preferenza, in ipotesi) che si possa avere per l'Italia dei sapori (sull'Italia della pittura, o della architettura, o delle spiagge, o della moda, o del canto, o delle tradizioni, o della poesia, o "dell'Ave Maria", giusto per rammentare la vitalità anche del turismo religioso che non può risolversi in una scampagnata). 